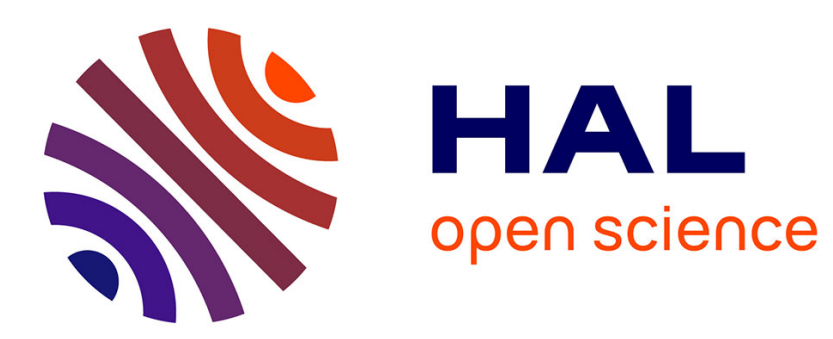

\title{
Ici et ailleurs, maintenant: scénographies de la présence dans les théâtres virtuels
}

\author{
Clarisse Bardiot
}

\section{To cite this version:}

Clarisse Bardiot. Ici et ailleurs, maintenant: scénographies de la présence dans les théâtres virtuels. Jean-Marc Larrue. Théâtre et intermédialité, Presses universitaires du Septentrion, pp.207-222, 2015, 10.4000/books.septentrion.8175 . hal-02336162

\section{HAL Id: hal-02336162 https://hal.science/hal-02336162}

Submitted on 28 Oct 2019

HAL is a multi-disciplinary open access archive for the deposit and dissemination of scientific research documents, whether they are published or not. The documents may come from teaching and research institutions in France or abroad, or from public or private research centers.
L'archive ouverte pluridisciplinaire HAL, est destinée au dépôt et à la diffusion de documents scientifiques de niveau recherche, publiés ou non, émanant des établissements d'enseignement et de recherche français ou étrangers, des laboratoires publics ou privés. 


\title{
Ici et ailleurs, maintenant : scénographies de la présence dans
}

\author{
les théâtres virtuels
}

\section{Clarisse Bardiot}

Parmi la multiplicité des formes qu'a pu prendre le théâtre, un seul paramètre ne varie pas : la co-présence physique des acteurs et des spectateurs, laquelle conférerait au spectacle son caractère « vivant ». La co-présence définit la réunion en un même lieu et un même temps des acteurs et des spectateurs. Sur cette base, toute scénographie théâtrale repose sur un système binaire : le lieu des acteurs et celui des spectateurs, le lieu de l'action et le lieu de l'écoute et du regard.

L'une des caractéristiques essentielles des théâtres virtuels est de comporter une interface - par exemple entre le comédien et l'environnement scénique, entre l'interprète et le spectateur, entre le plateau et le réseau. La présence de cette interface, c'est-à-dire d'un espace intermédiaire, rompt avec le modèle binaire de l'organisation de l'espace théâtral traditionnel. Par conséquent, elle remet profondément en question la manière de penser et concevoir la scénographie.

\section{Scénographies}

Les théâtres virtuels sont des " paysages de données ${ }^{1}$ » aux formes multiples et mouvantes : spectacles dans des environnements virtuels, dans des chats, plateaux équipés d'interfaces multiples, représentations à distance dans des lieux virtuels et/ou réels... L’espace scénique des théâtres virtuels n'est pas donné a priori. Il est en perpétuelle actualisation et nécessite, pour y avoir accès, une interface : espace intermédiaire, de transition, cette «membrane osmotique ${ }^{2} »$ permet aux différentes entités de communiquer entre elles, de rendre possible l'échange entre deux ou plusieurs

\footnotetext{
${ }^{1}$ «L'espace virtuel est fondamentalement un paysage de données, statiques ou dynamiques, avec des événements préprogrammés ou autonomes. Un rapport dramaturgique et scénographique de type nouveau paraît possible. » Philippe Quéau, Le virtuel : vertus et vertiges, Seyssel/Bry-sur-Marne, Champ Vallon/INA, coll. Milieux, 1993, p. 55.

${ }^{2}$ Paul Virilio, L'espace critique, Paris, Christian Bourgois, 1984, p. 18.
} 
systèmes hétérogènes. Espace instable où les frontières se font et se défont, l'interface sépare et réunit à la fois. Dans les théâtres virtuels, l'interface est la condition sine qua non de l'homogénéité du lieu de représentation, le nœud où peut s'organiser la rencontre entre l'acteur et le spectateur, entre la scène et la salle. Elle permet de définir la posture de l'acteur et du spectateur vis-à-vis de la scène, et donc les rapports scène-salle. Autrement dit, l'interface remplace le point de vue, critère normalement retenu pour analyser les dispositifs scéniques ${ }^{3}$. Trois grands types d'interface - l'image, le plateau et le réseau - permettent de définir trois types de scènes : les images-scènes, les scènes augmentées et les téléscènes.

\section{Les images-scènes}

Les images-scènes ont pour interface l'image numérique, celle-ci pouvant être diffusée sur tous types d'écrans, l'écran ultime étant la rétine. Elles se présentent sous forme d'installations interactives au sens large, depuis le CD-Rom jusqu'aux dispositifs immersifs. La plupart du temps, elles s'adressent à un seul spectateur, amené à agir dans/sur la représentation. Ces scènes individuelles, personnelles, suscitent des théâtres de l'intime, des théâtres pour soi.

Les images-scènes ont souvent pour contrepoint une version plateau. Le théâtre virtuel est alors accompagné d'un double, qui le précède ou lui succède, et qui a lieu sur une scène bien physique : le CD-Rom Puppet Motel prolonge le spectacle de Laurie Anderson, The Nerve Bible; Where Where There There Where, CD-Rom de Zoe Beloff, a été conçu lors des répétitions de House/Lights du Wooster Group; le jeu Afalud reprend et décline l'univers du spectacle Afasia, créés tous les deux par Marcel.lí Antúñez Roca; le site Internet $D Q$ anticipe l'opéra du même nom, mis en scène par la Fura dels Baus, etc.

Aussi, y compris lorsqu'elles ne sont pas dans le prolongement d'une version plateau, les images-scènes évoquent souvent les conventions de l'espace théâtral, gardant en mémoire l'espace du plateau, comme une trace rémanente de leur modèle. Une image fixe dont les limites sont celles de l'écran, une esquisse de perspective suffisent à rappeler la scène théâtrale à l'italienne. De même, le fond noir, que l'on pourrait penser neutre ou signifiant l'absence d'espace, suggère la profondeur de la cage de scène : les

\footnotetext{
${ }^{3}$ Cf. Étienne Souriau, "Le Cube et la sphère » (1948) in Architecture et dramaturgie, Paris, Éditions d'Aujourd'hui, coll. Les Introuvables, 1980 ; Jacques Polieri, Scénographie. Théâtre, cinéma, télévision, Paris, Jean-Michel Place, 1990 [1963] (réédition revue, corrigée et augmentée) ; Marcel Freydefont, « lieux scéniques » in Dictionnaire encyclopédique du théâtre, Paris, Bordas, 1991.
} 
visages-masques de Catherine Ikam et Louis Fléri, comme celui, féminin et asiatique, de l'installation Elle, ou encore les personnages de Luc Courchesne dans Salon des ombres, surgissent des profondeurs de l'espace numérique, comme s'ils étaient capturés par le faisceau d'un projecteur.

Pourtant, même si l'on est placé en face d'un écran qui nous rappelle tout de la fenêtre albertienne, ce n'est pas tant dans le dispositif frontal qu'il faudrait chercher un équivalent que dans les scènes éclatées ou panoramisantes, propres à susciter un déplacement du spectateur. En effet, l'espace représenté n'est pas celui de la perspective renaissante, liant tous les objets entre eux. Dans les images-scènes, on est face à une accumulation d'objets isolés, l'unité étant donnée dans le parcours, dans le cheminement du spectateur qui permet de tisser un lien entre des espaces dispersés ou des objets indépendants les uns des autres. C'est un espace à parcourir, une déambulation qui nous éloigne de la scène à l'italienne pour nous rapprocher de l'espace éclaté ou de l'espace à arpenter du théâtre de rue ou encore des mansions du théâtre du Moyen Age.

Les images-scènes sont souvent tentées par l'immersion ${ }^{4}$ du spectateur dans le dispositif : ce dernier est alors au centre de la représentation, au milieu de l'image. Ainsi Agnes Hegedüs, pour Memory Theater VR, place le spectateur au centre d'un panorama interactif, en référence au théâtre de la mémoire imaginé par Giulio Camillo. Dans Salon des ombres, installation présentée par Luc Courchesne comme un « théâtre vidéo interactif», le spectateur est au milieu d'un cercle défini par quatre pupitres, surmontés de quatre plaques de verre sur lesquelles sont projetées les images de quatre personnages qui donnent ainsi l'impression de flotter dans l'espace. Si ces recherches ont souvent été analysées en prenant comme arrière plan esthétique et théorique le cinéma, elles n'en sont pas moins fortement tributaires du spectacle vivant et du happening. Dès le début, les recherches sur le «cinéma élargi » sont l'occasion de renouer des liens entre spectacle et cinéma, notamment dans la lignée des futuristes ${ }^{5}$. Ainsi, c'est en termes d'espace théâtral, de scène ${ }^{6}$, qu'il convient d'analyser les dispositifs d'immersion actuels. Les $\mathrm{CAVE}^{7}$ en particulier sont conçus comme des

\footnotetext{
${ }^{4}$ Sur l'immersion, cf. Olivier Grau, Virtual Art. From Illusion to Immersion, Cambridge (Mass.), MIT Press, 2003.

${ }^{5}$ A ce propos, cf. Dominique Noguez, Éloge du cinéma expérimental, Paris, MNAM/Centre Pompidou, 1979.

${ }^{6}$ Tout au long de son article consacré à Jeffrey Shaw, Anne-Marie Duguet emploie le terme « scène » pour désigner les espaces conçus par ce dernier. Cf. « Du cinéma élargi à la réalité virtuelle. Jeffrey Shaw " in Anne-Marie Duguet, Déjouer l'image. Créations électroniques et numériques, Nîmes, Jacqueline Chambon, 2002, p. 119-172.

${ }^{7}$ CAVE signifie Cave Automatic Virtual Environment. Il s'agit d'un espace cubique dont les parois sont
} 
hybrides de performance et d'objet à explorer. Il ne s'agit plus seulement d'un écran devant lequel se trouve le spectateur, mais d'un espace physique combiné à un espace numérique dans lequel il est immergé tandis qu'il fait émerger les images. L'espace numérique devient spectacle.

Ainsi, l'espace est partagé, non plus entre des acteurs et un public, mais entre un lieu physique et un lieu numérique. En effet, les images-scènes favorisant un rapport intimiste, « one to one », du spectateur à la représentation, le rapport entre espace physique et espace numérique n'y est pas un rapport scène-salle. Celle-ci s'est fondue dans la scène, dissoute dans le lieu de l'action. Le lieu d'où l'on regarde - l'espace du spectateur - est devenu le lieu où l'on regarde et où l'on joue, comme le stigmatise l'attitude des spectateurs de Artificial Changellings, une installation de Toni Dove : ils s'agitent, semblent être investis d'un personnage, au point de se détourner de l'écran disposé sur l'un des murs de la salle (où sont projetées différentes séquences narratives dont ils contrôlent l'enchaînement par leurs déplacements), puis s'immobilisent, regardent à nouveau l'image. Parfois la salle subsiste peu ou prou, rejetée à la périphérie comme ces chaises le long des murs de la pièce où a été installée Artificial Changelings, ou encore derrière l'épaule du spectateur par-dessus laquelle on regarde Puppet Motel.

\section{Les scènes augmentées}

Dans les scènes augmentées, de la même manière que dans la « réalité augmentée », il y a adjonction, superposition d'un espace virtuel à un espace réel. Une scène augmentée est un plateau que l'on a interfacé avec différents objets numériques qui transforment la nature de l'espace scénique conventionnel. Dans les scènes augmentées, l'acteur, plus rarement un membre du public, manipule l'interface. Au sein des scènes augmentées, il est possible de distinguer deux démarches principales : la « scène-image » et la « scèneradiaire $»$.

La scène-image est la conversion de la scène en image, comme si la scène et l'écran se superposaient jusqu'à se confondre, en un paroxysme des « écrans sur la scène ${ }^{8}$ ». La projection d'images sur un tulle en avant scène, l'œil devant traverser l'écran pour percevoir ce qui a lieu sur le plateau, crée un espace ambigu, entre $2 \mathrm{D}$ et 3D. C'est le procédé le plus simple pour tenter de faire passer l'image projetée de la deuxième à la troisième dimension en créant l'illusion de la profondeur. Il a notamment été utilisé par

recouvertes d'écrans sur 3 à 6 faces selon les modèles. Des lunettes permettent de voir les images projetées en 3D. La diffusion du son est spatialisée, renforçant ainsi le sentiment d'immersion.

${ }^{8}$ Cf. Béatrice Picon-Vallin (dir.), Les écrans sur la scène, Lausanne, L'Âge d'Homme, coll. Th XX, 1998. 
Cunningham dans Biped. Autre exemple : ce procédé permet à Adrien Mondot de jongler avec des balles réelles et des balles virtuelles dans Convergence 1.0.

Plus spectaculaire, et liée à une histoire du théâtre âgée de plusieurs siècles, est la création d'images en 3D dans lesquelles sont immergés les acteurs, grâce à divers stratagèmes optiques, à des machines de vision, l'illusion étant d'autant plus parfaite que le spectateur ne revêt aucune prothèse visuelle. Ainsi, la projection d'une image sur un miroir sans tain placés entre les acteurs et le public donne l'impression que l'image «flotte » derrière le miroir et qu'acteurs et images partagent le même espace. Ce système était très utilisé au XIX ${ }^{\mathrm{e}}$ siècle pour faire apparaître des spectres. Il connaît un renouveau important aujourd'hui, par exemple dans les mises en scène de Victor Pilon et Michel Lemieux 9 . Des expériences avec des lunettes polarisantes sont également menées : George Coates en 1997 pour 20/20 Blake, Bob Wilson en 1998 pour un opéra de Philip Glass, Monsters of Grace, La Fura dels Baus pour Obs en 2000... Pour Mark Reaney, scénographe américain, «le théâtre est la première machine de réalité virtuelle $^{10} \gg$. Il se lance au début des années 1990 dans une série d'expérimentations où des images en 3D sont manipulées en temps réel en régie et regardées avec des lunettes polarisantes. En 1996, pour Wings, il va encore plus loin en munissant les spectateurs de casques $\mathrm{HMD}^{11}$ : ces derniers voient simultanément l'action scénique (sur le plateau sont également projetées des images) et les images projetées sur les écrans du casque.

Dès lors qu'elles sont vues en 3D, les images numériques utilisées dans ces spectacles sont des espaces pénétrables. Animées sur scène ou en régie, elles sont habitées par des acteurs. Il ne s'agit plus pour ces derniers de se caler le plus précisément possible sur le flux des images pour rendre ce dialogue plausible - comme le font avec virtuosité les danseurs de Montalvo et Hervieu ou encore les acteurs de Dumb Type - mais d'agir directement sur le comportement des images. Celles-ci n'obéissent plus à un flux invariable, celui du film, mais à la temporalité de la représentation. Le propos est décalé de l'écran à l'espace, de l'image à l'environnement scénique.

Dans la scène-radiaire, les éléments scéniques (images, sons, lumières, robots,

\footnotetext{
${ }^{9}$ À ce propos, cf. Frédéric Maurin, « Sans support ni surface ? Les images émancipées de 4D Art », Jeu : revue de théâtre, $\mathrm{n}^{\circ}$ 108, septembre 2003, p. 106-111 et Hélène Jacques, «Dans la Caverne d'Anima. Entretien avec Michel Lemieux et Victor Pilon », ibid., p. 99-105.

${ }^{10}$ Mark Reaney, «Virtual Reality on Stage », VR World, vol. 3, n 3, mai-juin 1995, p. 28.

${ }^{11}$ Le Head Mounted Display (HMD) est un casque de vision qui permet une immersion visuelle et parfois sonore dans des environnements virtuels. Inventé par Ivan Sutherland en 1967-68 au MIT, il comporte des capteurs de position permettant l'intégration des données relatives aux mouvements de tête de l'utilisateur ainsi qu'à la direction de son regard dans la construction dynamique de l'environnement virtuel. Certains dispositifs comportent des écouteurs et permettent de diffuser du son.
} 
accessoires, etc.) sont organisés soit en fonction du plateau soit en fonction de l'acteur. L'espace n'est pas unifié. Il est composé d'une multitude de zones dont on peut modifier les paramètres à chaque instant, d'une accumulation d'objets manipulables à distance par le comédien. Finalement, on ne représente plus l'espace scénique par un plan ou une coupe, mais par un circuit électronique schématisant les relations qui peuvent s'établir entre les différentes zones, entre les différents objets. De nombreuses recherches sont en cours dans ce domaine. Par exemple, la compagnie catalane Konic Thtr a commencé en 2005 un programme de recherche intitulé Escena augmentada. Ce projet repose sur la conception et le développement d'outils spécifiques : dispositifs de captation sans fil, traitement en temps réel de l'information, vision par ordinateur, etc. Cette scène augmentée doit notamment permettre une écriture chorégraphique non linéaire, basée sur un dialogue entre les danseurs et l'environnement scénique. Nou I_D Ópera electrónica para danza y $v_{0 z} z^{12}$ est une première étape vers la réalisation de cette scène augmentée. On est ici très proche des réflexions actuelles sur l'intelligence ambiante $^{13}$ : «Une ambient intelligence est un environnement transparent où sont impliquées informatique, technologie de réseautage avancées et interfaces spécifiques. Elle est consciente des caractéristiques spécifique de la présence humaine et des différentes personnalités, prend en charge les besoins et est capable de répondre intelligemment à des indications orales ou gestuelles, et peut même participer à un dialogue intelligent ${ }^{14}$. »

Dans une organisation en fonction du plateau, l'espace de jeu est équipé de toute une série de capteurs dont les champs d'action le recouvrent complètement. Ainsi, le logiciel Eyesweb, comme les dispositifs de Flavia Sparacino et de Robb Lovell, quadrillent l'espace avec des caméras. Dans cette configuration, l'acteur ne revêt aucun capteur, aucune prothèse, aucun instrument de contrôle spécifique. Son corps en mouvement, parfois la voix, lui permettent de manipuler les objets numériques. L'espace est défini en une zone active - la scène - et en une zone non active - la salle.

L'alternative à l'équipement du plateau, ce sont les «technologies embarquées ». Les capteurs ne sont plus répartis dans l'espace scénique, afin de le délimiter, mais sont

\footnotetext{
${ }^{12}$ [Notre traduction] Nou I_D, Opéra électronique pour danse et voix.

${ }^{13}$ Le terme désigne un environnement intelligent interactif, version française de ambient intelligence.

${ }^{14}$ [Notre traduction] «Ambient Intelligence implies a seamless environment of computing, advanced networking technology and specific interfaces. It is aware of the specific characteristics of human presence and personalities, takes care of needs and is capable of responding intelligently to spoken or gestured indications of desire, and even can engage in intelligent dialogue. » Giuseppe Riva et al. (dir.), Ambient Intelligence : the Evolution of Technology, Communication and Cognition Towards the Future of Human-Computer Interaction, Amsterdam/Fairfax, IOS Press, 2005, p. V.
} 
directement placés sur le corps des acteurs. Le costume lui-même devient l'interface qui permet de dialoguer avec le système, permettant de contrôler, en tout ou en partie, les éléments scéniques. Les exemples les plus représentatifs de cela sont les exosquelettes de Stelarc ou de Marcel.lí Antúñez Roca. Ancien membre de La Fura dels Baus, ce dernier a conçu un appareillage spécifique pour Afasia, en 1998. Dans cette « performance interactive », grâce à une interface créée spécifiquement pour l'acteur un exosquelette ${ }^{15}$ truffé d'interrupteurs au mercure, de potentiomètres et de capteurs -, tous les mouvements des coudes, des genoux, ainsi que la position des doigts, sont captés en temps réel, transmis par une liaison radio, et traduits en ordres à l'ordinateur. En se conformant à une gestuelle précise, Marcel.lí Antúñez maîtrise les objets numériques de ce plateau technologique, décuplant l'action de son corps, pilotant à distance tous les éléments composant le spectacle : images projetées sur l'écran en fond de scène, robots musicaux et environnement sonore.

Dans ce type de performance, l'espace scénique se construit à partir du corps et des actions du comédien. A tel point que pour Mark Coniglio, co-directeur de la compagnie américaine Troïka Ranch, l'acteur, l'image et le son qu'il manipule sont une seule entité $^{16}$. Le dispositif scénique est alors indissociable du comédien.

L'objectif de ce type d'interface demeure identique à celui des scènes augmentées dont les capteurs sont répartis sur le plateau : contrôler les différents objets et éléments de la scénographie à partir de l'acteur. Cependant la démarche est différente : il ne s'agit plus de délimiter une zone d'interaction dans laquelle l'acteur vient se placer, mais de partir d'informations recueillies directement sur le corps de l'acteur pour organiser et contrôler l'espace de jeu. L'unité de celui-ci ne provient plus de la répartition des capteurs dans l'espace, mais de la relation de ses différents éléments au corps de l'acteur, dans la limite de la longueur des câbles ou des ondes radio.

\section{Les téléscènes}

Les téléscènes sont des scènes à distance, des scènes en réseau. Ces «scènes à distance » peuvent être soit des lieux numériques, comme des forums ou des environnements virtuels disponibles sur Internet, soit des lieux réels, comme des plateaux de théâtre ou des cybercafés reliés par Internet, ou par un réseau créé pour l'occasion: ville virtuelle et ville réelle pour Can You See Me Now de Blast Theory,

${ }^{15}$ Un exosquelette est un squelette externe qui est placé sur le corps du comédien afin d'augmenter ses potentialités.

${ }^{16}$ Mark Coniglio, entretien avec Clarisse Bardiot, New York, septembre 2003. 
webcam filmant le plateau et dispositifs de chat dans certains spectacles de JeanFrançois Peyret, chats textuels ou visuels reliant acteurs et spectateurs répartis aux quatre coins du monde dans les représentations des compagnies The Hamnet Players et Desktop Theater. Agnès de Cayeux, dans ses différentes créations pour le réseau, s'inspire des chambres de discussion vidéo-chat du réseau Internet: «il s'agit d'interroger le réseau Internet, des usages que nous en avons à la " nature " que nous pouvons en pressentir. Le corps comme espace public, ce retrait radical de l'intime. Il s'agit aussi de rêver à une définition du réseau qui s'éloignerait radicalement d'un tout marchand et pornographique. L'utopie désignée. Ce réseau-là comme une préfiguration intelligente et sensible de notre propre évolution ${ }^{17}$. »

Les combinaisons de lieux numériques, hébergés sur des serveurs, avec des lieux réels, sont multiples. La scène et la salle ne sont plus alors des espaces physiques ou des espaces virtuels mais une combinaison des deux. Elles ne sont plus des entités circonscrites, mais éclatées, démultipliées, dispersées. Leur nature et leur taille varient au rythme des connexions.

Le réseau permet d'identifier un lieu de l'action, une scène. Cependant, l'identification d'un lieu du regard est beaucoup plus délicate, les spectateurs des téléscènes étant éparpillés dans des lieux éclatés. La scène est le réseau / le réseau est la scène. D’elle partent en étoile les connexions vers les acteurs lorsqu'ils sont téléprésents, mais surtout vers les spectateurs, on devrait dire vers chaque spectateur. Parfois représentée, la salle est le plus souvent remplacée par un accès à la scène, cet accès définissant les modalités du point de vue et de la relation aux acteurs et aux autres spectateurs. La séparation entre la scène et la salle n'est plus une question de répartition dans l'espace mais de l'attribution d'une posture, et finalement de règles, de codes. Le spectateur est défini par des paramètres qui font partie du déroulement de la représentation. On détermine la marge de manœuvre, la circulation, jusqu'aux modalités de figurations du spectateur sur la scène. Et ce sont bien ces attributions qui permettent de différencier un acteur d'un spectateur lorsqu'ils sont représentés par des avatars similaires, davantage que sa position dans l'espace, comme dans waitingforgodot.com, une adaptation de la pièce de Beckett pour le Palace par la compagnie Desktop Theater.

Ainsi, dans les téléscènes, le théâtre (au sens d'architecture) ne se définira pas tant en fonction de la nature des lieux qu'il juxtapose qu'en fonction de la configuration du réseau reliant les différentes composantes : acteurs, spectateurs, scène, salle. En effet, si

\footnotetext{
${ }^{17}$ Agnès de Cayeux, dossier de presse de In My Room, 2005.
} 
la notion de public est bien souvent évacuée des images-scènes - car elles suscitent la plupart du temps une relation intime de l'utilisateur avec le dispositif - elle ressurgit avec les téléscènes : où est le public et comment sa présence se manifeste-t-elle ? Faut-il représenter le public dans un environnement virtuel partagé, autrement dit en créant une salle virtuelle? Ou au contraire faut-il l'abstraire de la représentation et lui laisser simplement la possibilité de se connecter? Dans quelle mesure peut-il réagir ou participer ? Ce faisant, c'est dans la définition de ces paramètres que se joue la mise en scène de certains enjeux politiques d'Internet, entre contrôle du réseau et utopies collectives.

Finalement, ce qui est proposé dans les théâtres virtuels n'est plus le partage d'un même espace, et la disparition de la salle le montre, mais le partage d'un espace-temps, ici et ailleurs, maintenant. Ainsi, dans les téléscènes, le rendez-vous donné au spectateur n'est pas fixé en terme de lieu mais en terme d'horaire GMT. Dans les théâtres virtuels, l'enjeu n'est pas tant celui du temps réel que celui du temps privilégié du rendez-vous qui postule une rencontre, y compris avec des êtres numériques « enregistrés » comme les êtres mis en scène par Denis Marleau. La scéno-graphie, l'écriture de l'espace, est aussi écriture du temps. Cependant ce temps n'est pas tant défini par la représentation que par le spectateur et/ou l'acteur qui parcourt l'espace proposé. C'est un temps qui permet de déplier l'espace. Dans les théâtres virtuels, le temps s'articule comme espace. Le temps, celui du déplacement, de la variation, de la métamorphose de l'espace, s'est infiltré dans la configuration du lieu scénique.

\section{Présences}

L'une des caractéristiques communes aux multiples formes de théâtres virtuels est l'interface, laquelle introduit une médiation : les spectateurs et les acteurs se rencontrent et communiquent entre eux et avec l'environnement numérique par le biais d'artefacts. La médiation serait donc synonyme d'une perte de présence: les acteurs seraient absents aux spectateurs, et chaque spectateur serait absent aux autres spectateurs. Voire les spectateurs seraient absents des théâtres virtuels.

L'interface, en introduisant un espace intermédiaire, une zone de contact, change radicalement le rapport entre espace de la représentation et espace de l'écoute et du regard, entre le faire et le voir. Il y a une limite beaucoup moins tranchée entre les positions respectives de l'acteur et du spectateur, ce que certains ont appelé le 
« spectacteur ${ }^{18} »$. De nombreux auteurs, dont Lev Manovich ou encore Jean-Louis Weissberg («l'action sur l'image »), rappellent que voir et agir sont liés dès le début de l'adjonction de l'écran au calculateur ${ }^{19}$. L'action engendre une continuité entre les deux espaces, elle établit une relation entre deux entités maintenues traditionnellement à distance dans la culture occidentale.

Ainsi, l'interface permet d'établir un lien entre espace physique et espace numérique. À cause du scintillement des écrans, qui retient toute l'attention, ce lien a rarement été pris en compte. Pour prendre l'exemple le plus simple qui soit, le tapis de souris sur lequel se promène la main du spectateur des CD-Rom Puppet Motel ou Where Where There There Where est la correspondance dans le monde physique de la scène numérique, sorte de plateau miniature où se joue aussi la représentation. La continuité entre espace physique et espace numérique rapproche le spectateur de la scène, les lie l'un à l'autre dans une relation qui favorise un aller-retour constant entre immersion et mise à distance. Si bien que la coupure sémiotique entre la scène et la salle disparaît au profit d'une coupure entre espace physique et espace numérique, le rapport entre ces deux derniers n'étant pas un rapport scène-salle.

Dans les théâtres virtuels, faire et voir ne sont plus opposés, favorisant ainsi l'émergence de nouvelles postures de l'acteur et du spectateur. L'un et l'autre peuvent être considérés comme des « joueurs » (au sens de jouer une pièce) et il ne s'agirait pas tant de distinguer le voir du faire, que le geste amateur (le spectateur) du geste expert (l'acteur).

C'est ainsi que de nombreux spectacles sont déclinés en installations interactives dans lesquelles le spectateur vient prendre la place de l'acteur. Pour citer quelques exemples : Messa di voce de Golan Levin et Zachary Lieberman (2003); plusieurs spectacles et installations de Dumb Type ou encore Konic Thtr. Ce procédé permet au spectateur de mieux comprendre le jeu des technologies dans le spectacle présenté. L'acteur apparait comme un spécialiste habile, et est davantage à même de faire ressortir tout le potentiel dramaturgique du dispositif. Il demeure un professionnel de l'interprétation, tandis que

18 Voir Jean-Louis Weissberg, Présences à distance. Déplacement virtuel et réseaux numériques : pourquoi nous ne croyons plus à la télévision, Paris, L'Harmattan, 1999. Voir également: Jean-Louis Weissberg et Étienne Armand Amato, «Le Corps à l'épreuve de l'interactivité : interface, narrativité, gestualité », Anomalie Digital_arts «Interfaces », n 3, mars 2003, p. 41-51. Le terme « spect-acteur» a d'abord été employé par Augusto Boal dans les années 1960 pour désigner le spectateur qui monte sur scène dans le Théâtre de l'opprimé. Cependant, Jean-Louis Weissberg ne fait pas référence à Boal lorsqu'il emploie ce terme.

${ }^{19}$ Cf. Lev Manovich, The Language of New Media, Cambridge (Mass.), MIT Press, 2001; Weissberg Jean-Louis, Présences à distance, op. cit. 
le spectateur apparaît comme un amateur, plus ou moins entraîné. L'acteur est bien souvent convoqué dans la phase de conception du dispositif. Le spectateur l'investit dans un second temps. Cela implique pour l'acteur un apprentissage et une maîtrise de son corps spécifiques, bien au-delà des capacités développées par les spectateurs. Il ne faut pas sous-estimer ce point, car la réussite ou l'échec des théâtres virtuels dépend bien souvent de la capacité de l'acteur à s'adapter à ces nouvelles conditions de jeu.

Le film américain Being John Malkovich décrit la situation suivante: différentes personnes investissent successivement le corps de l'acteur John Malkovich qui interprète son propre rôle. Elles ont en général un rôle passif : elles voient le monde à travers ses yeux et pénètrent ses pensées les plus secrètes. Seuls le héros marionnettiste, qui ne se prénomme pas Craig ${ }^{20}$ par hasard, et parfois sa femme peuvent le manipuler et le transformer en pantin, jusqu'à faire faire à l'acteur des actions qu'il désapprouve. Habité par Craig, John Malkovich ne peut s'empêcher d'exécuter les quatre volontés de celui-ci. Tout comme les hommes et les femmes qui investissent le corps et l'esprit de John Malkovich, les joueurs des théâtres virtuels se coulent dans la peau d'acteurs virtuels, de personnages numériques, dont le corps de données est préexistant.

Comme le rappelle Robert Abirached dans La Crise du personnage dans le théâtre moderne, persona en latin signifie masque. La conséquence de cette filiation avec le masque, c'est qu' « oublié et s'oubliant derrière son personnage, l'acteur perd alors son identité pour devenir tout entier un idéogramme en mouvement ${ }^{21}$. » Définir le personnage comme un «idéogramme en mouvement» évoque l'origine grecque de personnage (kharactêr : signe gravé, empreinte), qui a donné character en anglais et caractère en français. Character signifie à la fois le personnage et la lettre, rappelant par là même que le personnage est inscrit dans un texte. Le personnage est « entre le mot et le corps, entre la puissance et l'acte, entre le songe et le réel ${ }^{22} »$. Être de langage en attente d'incarnation, il préexiste et subsiste à l'acteur, celui-ci n'offrant du personnage qu'une version possible. En mettant le vocabulaire au goût du jour, on pourrait dire des acteurs virtuels qu'ils sont entre le code et la vie, entre la puissance et l'actualisation, entre l'imaginaire et le réel. De même que le personnage, l'acteur virtuel est inscrit dans un texte, le code binaire de l'informatique ${ }^{23}$. Les acteurs virtuels ne sont donc pas tant

\footnotetext{
${ }^{20}$ Référence à Edward Gordon Craig, metteur en scène et théoricien défenseur d'une technique de jeu fondée sur la manipulation de marionnettes.

${ }^{21}$ Robert Abirached, La Crise du personnage dans le théâtre moderne, Paris, Gallimard, 1994 [1978], p. 19.

${ }^{22}$ Ibid., p. 7.

${ }^{23}$ Isabelle Rieusset-Lemarié développe une analyse assez proche à propos de l'avatar. Cf. Isabelle Rieusset-Lemarié, "Au-delà du "Corps de substitution", l'avatar caractère d'une écriture interactive »,
} 
des acteurs que des personnages dotés d'autonomie, à des degrés divers, et qui se définissent dans le rapport instauré avec le joueur qui interagit avec eux. Pour rappeler leur ancrage dans l'écriture - le code -, nous les appellerons « caractères numériques ». Le joueur est représenté dans les théâtres virtuels, que ce soit visuellement, comme avec l'avatar, ou parce que son déplacement entraîne une modification de l'image, du son, des lumières, etc. De fait, ces différents objets avec lesquels le joueur entre en relation peuvent également être considérés comme des caractères numériques.

Lieu de convergence du voir et du faire, du spectateur et de l'acteur, les caractères numériques des théâtres virtuels ont déjà un corps, fait de données numériques, parfois issues, grâce à des procédés de capture, d'un corps de chair. Les caractères numériques ne sont plus en attente d'un auteur mais d'un joueur qui leur permette d'être joués. Dans les théâtres virtuels, ce n'est plus le personnage qui s'incarne dans un acteur, mais le spectateur et/ou l'acteur qui s'incarne(nt) dans le personnage.

L'incarnation dans un être virtuel demande au joueur un engagement physique minimum, ne serait-ce que le maniement de la souris, y compris lorsque le pointeur n'est pas représenté à l'écran. C'est le geste et son effectivité qui vont produire à la fois ce sentiment de présence et sa matérialisation dans les théâtres virtuels : le surgissement du geste s'inscrit dans le présent; il est acte de présence. Précisons que le geste n'est pas uniquement un mouvement $\mathrm{du}$ corps. Nous considérons comme geste toute manifestation physique de la part du joueur - par exemple l'émission d'un son (geste sonore). Cet aspect est important, en particulier pour les interfaces à reconnaissance vocale.

Par ailleurs, parce qu'il doit revêtir le masque du caractère numérique, le joueur doit préciser sa gestuelle. Le masque a en effet cette faculté d' «organiser [le] corps et [le] $\mathrm{jeu}^{24} »$. Jacques Lecoq, dans les exercices de jeu masqué qu'il propose dans son école, rappelle que « nous pouvons nous laisser guider par la forme elle-même, telle qu'elle est proposée par la structure du masque. Celui-ci devient alors comme un véhicule, entraînant tout le corps dans l'espace, dans des mouvements particuliers qui font apparaître le personnage $\mathrm{e}^{25}$. T Tout comme le masque, le caractère numérique implique une gestuelle que le joueur doit trouver et s'approprier.

\footnotetext{
Anomalie digital_arts, Du Corps à l'avatar, $\mathrm{n}^{\circ}$ 1, oct. 2000.

${ }^{24}$ Jacques Lecoq (dir.), Le Théâtre du geste. Mimes et acteurs, Paris, Bordas, 1987, p. 115.

${ }^{25}$ Jacques Lecoq, Jean-Gabriel Carasso et Jean-Claude Lallias, Le corps poétique. Un enseignement de la création théâtrale, Arles, Actes Sud, coll. Papiers, 1997, p. 66.
} 
Le joueur s'inscrit dans ces dispositifs davantage en terme de présence que d'action. En effet, l'implication du joueur évolue de l'interactivité vers la prise en compte de sa présence, autrement dit d'une action consciente vers une action involontaire. Il n'interagit pas avec une interface selon un mode d'emploi donné ou à deviner : sa présence - parfois sa présence à distance - suffit à influer/influencer la scène. Ainsi, dans la version installation de L'Assemblée de Bill Vorn et Louis-Philippe Demers, les actions des robots sont déclenchées par l'intrusion d'un joueur au sein du dispositif. En fonction de son comportement, de ses déplacements, de ses mouvements, le jugement des machines sur «le dernier homme», comme le précise le programme, évoluera différemment. Dans cette évolution, l'action du joueur ne peut être directive : il n'a pas de «mode d'emploi », et l'on serait bien en peine de lui en donner un, puisque les robots, grâce à des programmes issus de l'intelligence artificielle, simulent des phénomènes de foule. Ils ne répondent pas à des actions précises, mais à un enchaînement de comportements de la part du joueur.

Hans-Thies Lehmann, dans son ouvrage sur le théâtre post-dramatique, constate que « la catégorie adéquate pour le nouveau théâtre n'est point l'action, mais l'état et la situation. Le théâtre nie intentionnellement la possibilité de "développer une fable", ou, en tout cas, la relègue au second $\operatorname{plan}^{26}$. » L'une des caractéristiques du théâtre postdramatique est dans le passage de la représentation à la présentation, passage qui réside dans la mise en valeur du processus et de l'expérience. Privilégier la situation sur l'action, c'est renouer d'une autre manière avec la présence. Les théâtres virtuels sont des théâtres du temps présent, le temps de la situation qui suppose l'implication de toutes les parties, le temps du processus qui a lieu, le temps du surgissement de la parole.

L'écriture propre aux théâtre virtuels place l'expérience du joueur, qu'il soit acteur (geste expert) ou spectateur (geste amateur), au centre du dispositif : écrire pour les théâtres virtuels, c'est avant tout écrire pour le joueur. Si représentation il y a, ce n'est ni de la réalité, ni d'un texte ou d'une action antérieurs au spectacle qui les rendrait à nouveau présents, mais du joueur dans le dispositif lui-même. Cette représentation prend en compte deux aspects: la représentation du joueur dans le système (par exemple la façon dont il apparaît : avatar, capture vidéo, texte, etc.) et la représentation du joueur pour le système (par exemple, modélisation de ses actions/réactions afin que le système génère la suite de la fable). Écrire le joueur, c'est non seulement lui donner

\footnotetext{
${ }^{26}$ Hans-Thies Lehmann, Le Théâtre postdramatique, Paris, L’Arche, 2002, p. 104.
} 
un rôle à jouer, mais aussi définir les modalités de sa présence et du jeu. Autrement dit, dans les théâtres virtuels, la question de la représentation ne se pose pas par rapport à un texte ou à une réalité préexistants qu'il s'agirait de re-présenter, mais par rapport à la représentation du joueur dans le dispositif lui-même.

\section{Conclusion}

L'hybridation entre espace physique et espace numérique permet de redéfinir la scénographie comme une enveloppe parcourue par des flux. L'enveloppe englobe à la fois la scène et la salle. Elle n'est pas une forme fixe, mais un contenant souple, mouvant, fluctuant au gré des changements et des variations du contenu. La souplesse de l'enveloppe, sa ductilité sont nécessaires, car les espaces scéniques des théâtres virtuels sont parcourus par des flux qui provoquent des variations de l'espace. Ces flux, ces mouvements sont de deux sortes : mouvement du joueur, qui parcourt la scène, l'explore, la modifie, mais aussi mouvement de la scène, en perpétuelle mutation, transformation, actualisation. La vision globale que propose la scène à l'italienne est remplacée par une vision subjective et parcellaire, dépendante de la manipulation du spectateur et/ou de l'acteur.

Cette versatilité de l'espace numérique, Marcos Novak l'a utilisée pour définir l'«architecture liquide» et la «transarchitecture »: «Le cyberespace est liquide. Cyberespace liquide, architecture liquide, villes liquides. L'architecture liquide est plus que l'architecture cinétique, que l'architecture robotique, c'est une architecture de parties fixes et de liens variables. [...] L'architecture liquide est une architecture dont la forme est contingente des intérêts de son propriétaire ${ }^{27}$.» Plutôt que scénographie liquide, qui a tendance à occulter la mobilité du spectateur, nous préférons employer le terme de scénographie moirée pour définir les espaces scéniques des théâtres virtuels. La moire, ce tissu aux reflets mouvants, chatoyants, exprime bien les variations et changements constants de l'espace et du lieu d'où l'on regarde. Dans une scénographie moirée, l'espace est lié au spectateur et change en fonction de sa position tout comme il est animé de son propre flux. Ils sont indissociables et pourtant dotés d'une autonomie spécifique.

Le flux et l'enveloppe sont caractéristiques non seulement de la scénographie, mais aussi de l'écriture, de l'acteur et du spectateur ${ }^{28}$. Toutes les composantes des théâtres

\footnotetext{
${ }^{27}$ Marcos Novak, «Liquid Architectures in Cyberspace » in Michael L. Benedikt (dir.), Cyberspace : First Steps, Cambridge (Mass.), MIT Press, 1991, p. 249.

${ }^{28}$ Cf. Clarisse Bardiot, Les Théâtres virtuels, thèse de doctorat, Université Paris III - Sorbonne Nouvelle,
} 
virtuels sont concernées. De fait, nous considérons que le flux et l'enveloppe définissent ce que nous appellerons une "dramaturgie du paramorphe ». Le terme paramorphe est un anglicisme. Nous l'empruntons à Mark Goulthorpe, architecte fondateur du groupe deCOi. Ce terme désigne « une figure qui peut varier dans ses formes tout en conservant ses propriétés essentielles ${ }^{29} »$.

La dramaturgie du paramorphe ne définit pas un théâtre sans forme, un théâtre informe, mais un théâtre des formes possibles : un théâtre virtuel, au sens étymologique. Dans la dramaturgie du paramorphe, une enveloppe est traversée/informée par des flux qui la définissent en retour. Rien de figé, mais un processus en mouvement, une métamorphose ininterrompue, une transformation transitoire et malléable. Théâtres du processus, de la métamorphose et de l'expérience, les théâtres virtuels reposent sur la détermination des variables qui composent l'acte théâtral.

Ainsi, tout comme le faire et le voir, l'actif et le passif, le physique et le virtuel, la scène et la salle, ne peuvent être strictement opposés et renvoyés dos à dos dans une logique binaire, tout comme ces couples se combinent et se contaminent à l'infini, il n'est plus possible d'opposer la présence à l'absence dans les théâtres virtuels ${ }^{30}$. Le spectateur et l'acteur peuvent être "présents à distance », non seulement dans les téléscènes mais aussi dans les images-scènes et dans les scènes augmentées. Selon les interfaces et le type de caractère, cette présence se déploie dans une gamme composée de différents degrés. Le face à face physique entre l'acteur et le spectateur peut alors être considéré comme l'un des degrés de cette gamme.

$2005,537 \mathrm{f}$.

${ }^{29}$ Josef Albers, Architectures non standard, Catalogue d'exposition, Paris, Centre Pompidou, 2003, p. 76.

${ }^{30} \mathrm{~N}$. Katherine Hayles développe une analyse semblable pour les textes littéraires et les corps humains. Elle propose de remplacer le couple présence/absence par celui de structure/aléatoire. Cf. N. Katherine Hayles, How We Became Posthuman. Virtual Bodies in Cybernetics, Literature and Informatics, Chicago, University of Chicago Press, 1999. 


\section{Bibliographie}

ABIRACHED, Robert, La Crise du personnage dans le théâtre moderne, Paris, coll. Tel, Gallimard, 1994 [1978], 506 p.

Albers, Josef, Architectures non standard, Catalogue d'exposition, Paris, Centre Pompidou, 2003, 223 p.

BARDiot, Clarisse, Les Théâtres virtuels, thèse de doctorat, Université Paris III Sorbonne Nouvelle, 2005, 537 f.

Cayeux, Agnès de, Dossier de presse de In My Room, 2005.

Duguet, Anne-Marie, Déjouer l'image. Créations électroniques et numériques, Nîmes, Jacqueline Chambon, 2002, $221 \mathrm{p}$.

Freydefont, Marcel, Article «lieux scéniques » in Dictionnaire encyclopédique du théâtre, Paris, Bordas, 1991.

Grau, Olivier, Virtual Art. From Illusion to Immersion, Cambridge (Mass.), MIT Press, 2003, 416 p.

Hayles, N. Katherine, How We Became Posthuman. Virtual Bodies in Cybernetics. Literature and Informatics, Chicago, University of Chicago Press, 1999, 350 p.

JaCques, Hélène, « Dans la caverne d'Anima. Entretien avec Michel Lemieux et Victor Pilon », Jeu : revue de théâtre, $\mathrm{n}^{\circ}$ 108, septembre 2003, p. 99-105.

Lecoq, Jacques (dir.), Le Théâtre du geste. Mimes et acteurs, Paris, Bordas, 1987, 152 p.

Lecoq, Jacques, Jean-Gabriel Carasso et Jean-Claude Lallias, Le corps poétique : un enseignement de la création théâtrale, Arles, Actes Sud, coll. Papiers, 1997, 170 p.

Lehmann, Hans-Thies, Le Théâtre postdramatique, Paris, L’Arche, 2002, 307 p.

Manovich, Lev, The Language of New Media, Cambridge (Mass.), MIT Press, 2001, $354 \mathrm{p}$.

Maurin, Frédéric, "Sans support ni surface? Les images émancipées de 4D Art », Jeu : revue de théâtre, $\mathrm{n}^{\circ} 108$, septembre 2003, p. 106-111.

Noguez, Dominique, Éloge du cinéma expérimental, Paris, MNAM/Centre Pompidou, 1979, $381 \mathrm{p}$.

Novak, Marcos, «Liquid Architectures in Cyberspace », in Michael L. Benedikt (dir.), Cyberspace : First Steps, Cambridge (Mass.), MIT Press, 1991, p. 249.

Picon-Vallin, Béatrice (dir.), Les Écrans sur la scène. Tentations et résistances de la scène face aux images, Lausanne, L'Âge d'Homme, coll. Th XX, 1998, 343 p.

Polieri, Jacques, Scénographie. Théâtre, cinéma, télévision, Paris, Jean-Michel Place, 1990 [1963], $191 \mathrm{p}$. 
QuÉAu, Philippe, Le Virtuel: vertus et vertiges, Seyssel/Bry-sur-Marne, Champ Vallon/INA, coll. Milieux, 1993, 215 p.

Reaney, Mark, «Virtual Reality on Stage », VR World, vol. 3, n 3, mai-juin 1995, p. 28-31.

RieusSET-Lemarié, Isabelle, "Au-delà du "Corps de substitution", l'avatar caractère d'une écriture interactive », Anomalie digital_arts, Du Corps à l'avatar, n¹, oct. 2000. PAGE ?

Riva, Giuseppe (dir.), et al., Ambient Intelligence: the Evolution of Technology, Communication and Cognition Towards the Future of Human-Computer Interaction, Amsterdam/Fairfax, IOS Press, 2005, 293 p.

Souriau, Étienne, « Le Cube et la sphère (1948) » in Architecture et dramaturgie, Paris, Éditions d'Aujourd'hui, coll. Les Introuvables, 1980.

VIRILIO, Paul, L'Espace critique, Paris, Christian Bourgois, 1984, 187 p.

Weissberg, Jean-Louis, Présences à distance. Déplacement virtuel et réseaux numériques : pourquoi nous ne croyons plus à la télévision, Paris, L'Harmattan, 1999, $301 \mathrm{p}$.

WEISSBERG, Jean-Louis et Étienne Armand AмAто, «Le Corps à l'épreuve de l'interactivité : interface, narrativité, gestualité », Anomalie Digital_arts "Interfaces », $\mathrm{n}^{\circ} 3$, mars 2003, p. 41-51. 